\title{
Hormonale und pharmakologische Beeinflussung der Sauerstoffzehrung des Bluts.
}

\author{
V. Mitteilung. \\ Über die kombinierte Wirkung verschiedener Inkrete und Vegetativ- \\ nervengifte auf den Sauerstofferbrauch des Bluts. \\ Von \\ Eishichiro Tsukamoto. \\ (塚 本 然 七 郎) \\ (Aus der medizinischen KTinik ron Prof. Dr. T. Kato, \\ Reichsuniversität $\approx u$ Sendai.)
}

Über die Wirkung einzelner Inkrete (Adrenalin, Insulin, Thyreoglandol, Pituglandol, Testiglandol, Ovoglandol, Thymoglandol, Epiglandol, Paraglandol und Anteglandol) und vegetativer Gifte (Atropin, Pilokarpin, Physostigmin, Cholin und Ergotamin) auf die Sanerstoffatmung des Bluts ist in meinen früheren Arbeiten (II. und III. Mitteilung) ausführlich berichtet worden. Nun wurde in vorliegender Arbeit die Veränderung des Sauerstoffverbrauchs des Bluts in vitro bei kombinierter Anwendung einiger Inkrete und Vegetativnervengifte untersucht.

\section{Kombinierte Wirkung von Insulin und} Atropin.

Bekanntlich ist Atropin ein Vertreter der parasympathikuslähmenden Gifte, und nach Garrelon und San tenoise $\mathrm{D}^{\mathrm{D}}$ u. a. reagiert das parasympathische Nervensystem auf Insulinzufuhr mit einer stundenlang anlaltenden physiologischen Erregbarkeitssteigerung, so dass Insulin der parasympathikuserregenden Giftgruppe einzureihen ist. Dass Insulin und Atropin auf den Energieumsatz nicht nur des Gesamtorganismus, sondern auch des isolierten Gewebes in entrgegengesetztem Simne wirken, ist schon von einigen Autoren nachgewiesen worden.

1) Garrelon und Santeno ise, Compt. rend, Soc. Biol., 1924, 90, 470. 
Bei gleichzeitigem Zusat $z$ von Insulin und Atropin erzielte A h lg ren ${ }^{\text {") }}$ nach der Methylenblaumethode gegenseitige Hemmung inbezug auf die Gewebsatmung. Ferner bemerkte $\mathrm{H}_{\mathrm{olb}} \mathrm{ll},{ }^{3}$ dass in den Atropincersuchen Insulin die akzelerierende Wirkung des Atropins auf die Gewebsoxydation völlig aufhebt.

Ich habe den Einfluss des gleichzeitigen Zusatzes von Insulin und Atropin zum Blut auf die Sauerstoffzehrung der Blutzellen nach der schon in den früheren Mitteilnngen geschilderten Methode untersucht. Tab. 1 stellt die Ergebnisse einer Untersuchung dar, bei der grosse Dosen Insulin

\section{Tabelle 1.}

Der Einfluss ron Insulin $+A$ tropin auf den Sauerstoffrerbrauch des Blutes.

(1 $\mathrm{cm}$ Blut : 0,075 ccm Insulin $+0,075 \mathrm{mg}$ Atropin.)

I. A. = Blut mit Insulin +Atropin. K.=Kontrolle.

\begin{tabular}{|c|c|c|c|c|c|c|}
\hline \multirow{2}{*}{$\begin{array}{l}\text { Vers. } \\
\text { Nr. }\end{array}$} & \multirow{2}{*}{$\begin{array}{c}\text { Gewicht } \\
\text { (kg) }\end{array}$} & \multirow{2}{*}{ Geschlecht } & \multicolumn{4}{|c|}{$\mathrm{O}_{2}-$ Verbrauch (Vol. \%) nach } \\
\hline & & & \multicolumn{2}{|c|}{3 Std. } & 6 Std. & $9 \mathrm{Std}$. \\
\hline 1. & 1,6 & $\hat{b}$ & I. $A$. & 19,2 & $\begin{array}{l}27,5 \\
22,1\end{array}$ & $\begin{array}{l}39,7 \\
336\end{array}$ \\
\hline \multirow[t]{2}{*}{2.} & 1,6 & $?$ & I. $\mathrm{A}$. & 13,8 & 24,5 & 36,5 \\
\hline & & & $\mathrm{K}$. & 19,1 & 26,7 & 34,2 \\
\hline 3. & 1,9 & s & I. A. & 11,4 & 22,5 & 24,9 \\
\hline \multirow{2}{*}{58.} & 1,7 & 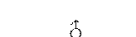 & $\begin{array}{l}\text { h. } \\
\text { I. A. }\end{array}$ & $\begin{array}{l}12,4 \\
16,5\end{array}$ & $\begin{array}{l}20,0 \\
24,2\end{array}$ & $\begin{array}{l}24,6 \\
32,6\end{array}$ \\
\hline & 1,1 & & & 14,3 & 20,5 & 27,1 \\
\hline \multirow{2}{*}{\multicolumn{3}{|c|}{ Durchschnittswert }} & 1. A. & 15,2 & 24,7 & 33,4 \\
\hline & & & $\mathrm{K}$. & 15,0 & 22,4 & 29,9 \\
\hline
\end{tabular}

und A tropin, nämlich 0,075 ecm Insulin Toronto (1 $\mathrm{EH} .=0,1 \mathrm{~cm})$ und 0,075 mg des letzteren (Atropin. sulfur. Merck) pro $1 \mathrm{ccm}$ defibrinierten Bluts, dem Blut zugesetzt und sein Sanerstoffverbranch dreistïndlich gemessen wurde.

Bei der Durchsicht obiger Tabelle findet man, dass der Sauerstoffkonsum des mit beiden Giften ver-

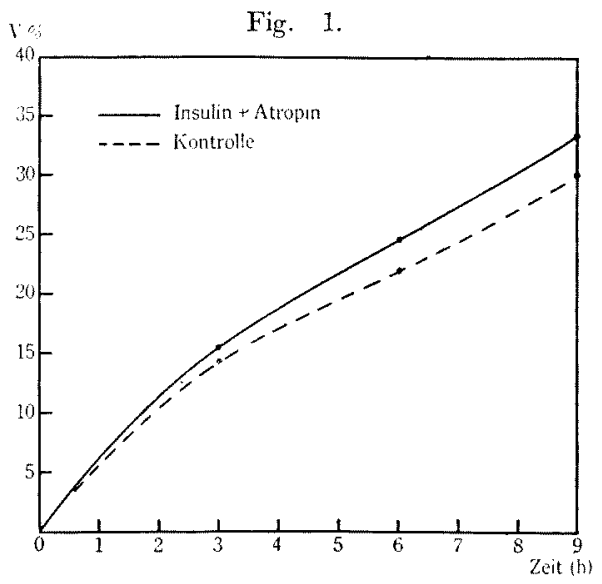

2) A hlgren, Kl. Wocbenschr., 1924, 667, Skand. Arch. f. Physiol., 1925, 46, 306.

3) Holboll, Skand. Arch. f. Physiol., 1926, 48, 225. 
setzten Blutes, gegenüber dem des Kontrollbluts, wenn auch nicht erheblich, doch verschicden ist und, wenn auch nicht ausnahmslos, im allgemeinen etwas grösser (Fig. 1). In meiner früheren Mitteilung habe ich gezeigt, dass auf den Sauerstoffverbrauch des Bluts in vitro Insulin in grösserer Dose deutlich, Atropin aber nur wenig fördernd wirkt.

Versetzt man $1 \mathrm{ccm}$ Blut mit einer Dose von Atropin und Insulin, die ungefähr der Hälfte derjenigen im vorigen Versuche entspricht, also mit $0,03 \mathrm{ccm}$ des letzteren und $0,03 \mathrm{mg}$ des ersteren, so ergibt sich fast dasselbe Resultat wie in Tab. 1 (Tab. 2).

\section{Tabelle 2.}

Der Einfluss ron Insulin + Atropin auf den Sanerstofferbrauch des Blutes.

(1 cen Blut : 0,03 cem Insulin $+0,03 \mathrm{mg}$ Atropin)

I. A.=Blut mit Insulin +Atropin. K.=Kontrolle.

\begin{tabular}{|c|c|c|c|c|c|c|}
\hline \multirow{2}{*}{$\begin{array}{l}\text { Vers. } \\
\text { Nr. }\end{array}$} & \multirow{2}{*}{$\begin{array}{c}\text { Gewicht } \\
(\mathrm{kg})\end{array}$} & \multirow{2}{*}{ Geschlecht } & \multicolumn{4}{|c|}{$\mathrm{O}_{2}$-Verbrauch (Vol. 9 ) nach } \\
\hline & & & \multicolumn{2}{|c|}{ s Std. } & $6 \mathrm{Std}$. & $9 \mathrm{Std}$. \\
\hline 4. & 1,7 & $\hat{\varepsilon}$ & $\underset{\mathrm{K}}{\mathrm{I}} .$. & $\begin{array}{l}27,5 \\
26,0\end{array}$ & $\begin{array}{l}33,5 \\
32,5\end{array}$ & $\begin{array}{l}39,8 \\
39,5\end{array}$ \\
\hline 5. & 1,5 & : & I. $A$. & $\begin{array}{l}10,7 \\
100\end{array}$ & 14,1 & 18,1 \\
\hline 6. & 1,8 & $\varepsilon$ & I. $\mathrm{A}$. & $\begin{array}{l}16,4 \\
13,1\end{array}$ & $\begin{array}{l}20,4 \\
17,5\end{array}$ & $\begin{array}{l}21,5 \\
20,6\end{array}$ \\
\hline 7. & $1, \mathrm{~S}$ & $\varsigma$ & ${ }_{\mathrm{K}}^{\mathrm{I}} . A$. & $\begin{array}{r}16,7 \\
9,3\end{array}$ & $\begin{array}{l}19,7 \\
15,3\end{array}$ & $\begin{array}{l}25,0 \\
22,7\end{array}$ \\
\hline Dur & aschnittsw & & $\begin{array}{l}\text { I. } A . \\
\mathrm{K} .\end{array}$ & $\begin{array}{l}17,8 \\
14,6\end{array}$ & $\begin{array}{l}22,0 \\
19,5\end{array}$ & $\begin{array}{l}26,1 \\
24,6\end{array}$ \\
\hline
\end{tabular}

Bei gleichzeitiger V'ersetzung von $1 \mathrm{ccm}$ Blut mit einer noch kleineren Dose von Insulin $(0,005 \mathrm{ccm})$ und Atropin $(0,005 \mathrm{mg})$ erhält man ein Ergebnis, das, wie in Tab. 3 u. Fig. 2 wiedergegeben, dem des Versuchs mit relativ grosser Dose ganz entgegengesetzt ist (vol. Tab. 1). Also ist der Sauerstoffkonsum des Bluts, wenn auch nur ein wenig, doch merkbar kleiner als der des Kontrollbluts. Bei Zusatz jedes der beiden Gifte allein zum Blut steigert Insulin in kleiner Menge die Blutatmung, während Atropin sie hemmt, wie ich in meiner früheren Mitteilung berichtet habe. Nun ist auch der vorliegende Versuch in dem Sinne ausgefallen, dass eine Kombination einer geringen Menge Insulin und A tropin den Sauerstoff verbrauch des Bluts hemmt. Eigentlich kommt der Atropinwirkung, wic bekannt, nach pharmakologischer Regel die Eigenschaft zu, die Wirkung der parasympathikusreizenden Gifte zu beseitigen. Dass dies anch beim Saucrstoffverbrauch des Bluts der Fall ist, ist eine sehr interessante Erscheinung. 
Tabelle 3.

Der Einfluss ron Insulin + Atropin auf den Sauerstofferbrauch des Blutes.

(1 ccm Blut : 0,005 ccm Insulin $+0,005 \mathrm{mg}$ Atropin)

I. A. = Blut mit Insulin + Atropin. K.=Kontrolle.

\begin{tabular}{|c|c|c|c|c|c|c|}
\hline \multirow{2}{*}{$\begin{array}{l}\text { Vers. } \\
\text { Nr. }\end{array}$} & \multirow{2}{*}{$\begin{array}{c}\text { Gewicht } \\
\text { (kg) }\end{array}$} & \multirow{2}{*}{ Geschlecht } & \multicolumn{4}{|c|}{$\mathrm{O}_{2}$-Verbrauch ( $\mathrm{Vol} \%$ ) nach } \\
\hline & & & \multicolumn{2}{|c|}{$3 \mathrm{Std}}$. & $6 \mathrm{Std}$ & $9 \mathrm{Std}$ \\
\hline 8. & 1,9 & $\mathrm{~s}$ & $\begin{array}{l}\text { I. A. } \\
\text { K. }\end{array}$ & $\begin{array}{l}14,8 \\
16,6\end{array}$ & $\begin{array}{l}24,8 \\
22,1\end{array}$ & $\begin{array}{l}30,0 \\
33,4\end{array}$ \\
\hline 9 . & 1,6 & 宇 & I. A & $\begin{array}{l}35,1 \\
355\end{array}$ & $\begin{array}{l}38,6 \\
394\end{array}$ & 41,1 \\
\hline 10. & 2,0 & 3 & $\mathrm{I} A$ & $\begin{array}{l}19,5 \\
202\end{array}$ & $\begin{array}{r}23,4 \\
251\end{array}$ & $\begin{array}{r}29,9 \\
309\end{array}$ \\
\hline 11. & 1,5 & $\overrightarrow{7}$ & $\mathrm{I}, \mathrm{A}$ & 12,1 & 17,8 & 21,2 \\
\hline \multicolumn{3}{|c|}{ Durchschnittswert } & $\begin{array}{l}\text { I. A } \\
\text { K. }\end{array}$ & $\begin{array}{l}20,6 \\
217\end{array}$ & $\begin{array}{l}26,1 \\
26,7\end{array}$ & $\begin{array}{l}30,6 \\
33,2\end{array}$ \\
\hline
\end{tabular}

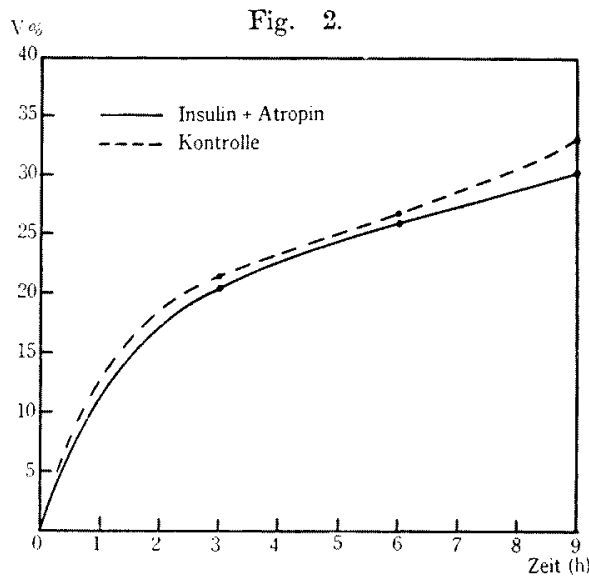

Zusammenfassung: Bei gleichzeitigem Zusatz von Atropin und Insulin zum Blut ist ihre Wirkungsweise je nach ihrer Menge verschieden. Bei grösserer Dose beider Gifte wird die Blutatmung gefördert, aber bei einer kleinen gehemmt.

2. Kombinierte Wirkung von Insulin und Pilokarpin.

Es war öfters davon die Rede, dass Insulin als Parasympathikusgift auf die Sauerstoffitmung der Blutzellen immer fördernd wirkt und, wie in der vorigen Arbeit gesagt, erhölt Pilokarpin, auch ein Parasympathikusgift, in kleincrer Menge die Blutzellatmung, wenn es sich auch bei ciner grösseren gegen sie indifferent verhält. Um zu erkennen, was für ein Einfluss auf den Sanerstoffkonsum des Blnts ausgeübt wird, wenn man die beiden Gifte gleichzeitig dem Blute zusetzt, habe ich folgende Untersuchung ausgeführt. 


\section{'Tabelle 4.}

Der Einfluss von Insulin + Pilokarpin auf den Sauerstoffverbrauch des Blutes.

(1 cem Blut : 0,075 ccm Insulin +0,075 mg Pilokarpin)

I. P. = Blut mit Insulin + Pilokarpin. K.=Kontrolle.

\begin{tabular}{|c|c|c|c|c|c|c|}
\hline \multirow{2}{*}{$\begin{array}{l}\text { Vers. } \\
\text { Nr. }\end{array}$} & \multirow{2}{*}{$\begin{array}{c}\text { Gewicht } \\
(\mathrm{kg})\end{array}$} & \multirow{2}{*}{ Geschlecht } & \multicolumn{4}{|c|}{$\mathrm{O}_{2}$-Verbrauch (Vol. :ó) nach } \\
\hline & & & \multicolumn{2}{|c|}{3 Std. } & $6 \mathrm{Std}$ & $9 \mathrm{Std}$. \\
\hline 12. & 1,6 & ? & I. $P$ & 14,8 & 19,2 & 22,2 \\
\hline \multirow{2}{*}{13.} & 1,5 & 9 & $\begin{array}{l}\text { h. } \\
\text { I. } P \text {. }\end{array}$ & $\begin{array}{l}13,3 \\
11,7\end{array}$ & $\begin{array}{l}10,4 \\
12,3\end{array}$ & 18,6 \\
\hline & & & $\mathrm{K}$. & 8,1 & 11,7 & 16,9 \\
\hline \multirow[t]{2}{*}{14.} & 1,6 & ? & I. $\mathrm{P}$. & 18,4 & 26,3 & 87,8 \\
\hline & 75 & 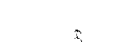 & K. & $\begin{array}{l}19,7 \\
161\end{array}$ & 21,7 & $\begin{array}{l}35,7 \\
308\end{array}$ \\
\hline 15. & 1,0 & 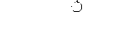 & $\mathrm{K}$. & $\begin{array}{l}10,1 \\
12,3\end{array}$ & 22,3 & 25,8 \\
\hline \multirow{2}{*}{\multicolumn{3}{|c|}{ Durchschnittswert }} & I. P. & 15,3 & 20,3 & 27,4 \\
\hline & & & $\mathrm{K}$. & 13,4 & 17,5 & 24,2 \\
\hline
\end{tabular}

Aus Tab. 4 geht hervor, dass der Sauerstoffverbrauch des Bluts durch gleichzeitigen Zusatz einer grösseren Menge von Insulin $(0,075 \mathrm{ccm})$ und Pilokarpin $(0,075 \mathrm{mg})$ zu 1 $\mathrm{ccm}$ Blut gesteigert wird (Fig. 3). Es stellt sich bei grösserer Dosierung beider Gifte nur die Wirkung des Insulins allein ein, da der Zusatz des Pilokarpins in

Fig. 3

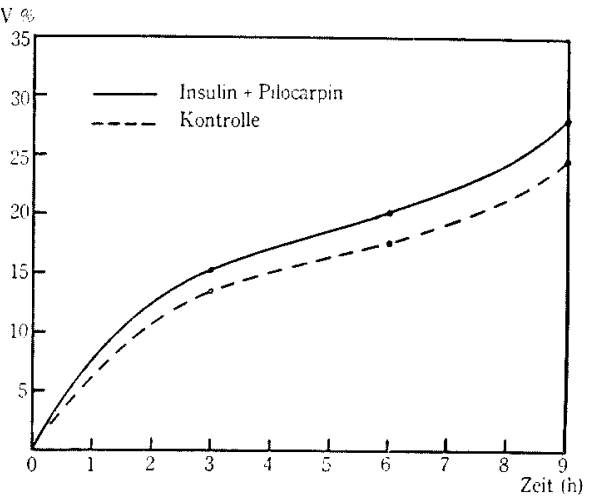
grösserer Menge an und für sich ohne Einfluss ist.

Bei der Versetzung des Bluts mit einer kleineren Menge beider Gifte (0,005 ccm Insulin, 0,0005 mg Pilokarpin : $1 \mathrm{ccm}$ Blut) zeigte sich, wie aus Tab. 5 ersichtlich, eine deutliche Förderung des Sauerstoffkonsums des Bluts.

Lässt man eine relativ kleine Dose Pilokarpin und Insulin jedes für sich einwirken, so wird die Blutzellatmung deutlich gesteigert, worauf ich in der vorhergehenden Arbeit eingegangen bin. Auch bei gleichzeitigem Zusatz beider Gifte erhöht sich die Blutatmung auffullend, welches Rosultat aber nicht die algebraische Summe der Wirkungren beider Gifte ist, wie die obige Tabelle zeigt. 


\section{Tabelle 5 .}

Der Einfluss von Insulin + Pilokarpin auf den Sauerstoffverbrauch des Blutes.

(1 $\mathrm{cm}$ Blut : 0,005 $\mathrm{ccm}$ Insulin $+0,005 \mathrm{mg}$ Pilokarpin)

I. P.=Blut mit Insulin +Pilokarpin. K.=Kontrolle.

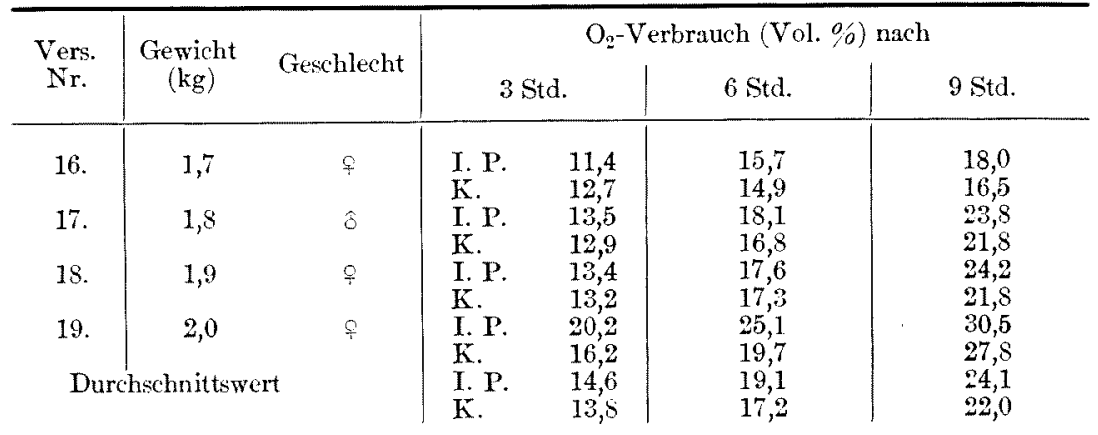

Nach Holbollib) wird in den Pilokarpininsulinversuchen, wo das Alkaloid zuerst zugesetzt worden ist, die fördernde Wirkung des Alkaloids geschwïcht, wïhrend in denjenigen Versuchen, wo Insulin zuerst zugesetzt worden ist, eine ausgesprochene Hemmung eintritt, da zwischen der Einwirkung des Insulins und des zu untersuchenden Pilokarpins auf die Gewebsatmung ein antagonistisches Verhältnis besteht. Aber nach meinem Versuchsergebnis wirken beide Gifte sowohl in grösseren als auch in kleineren Dosen niemals antagonistisch.

Zusammenfassung: Wird das Blut gleichzeitig mit Insulin und Pilokarpin versetzt, so wird sein Sauerstoffverbrauch immer gefördert.

\section{Kombinierte Wirkung von Adrenalin und Atropin.}

Es wurde in der zweiten Mittcilung erwähnt, dass Adrenalin nachweislich die Tendenz zeigt, auf die Sauerstoffatmung des Bluts in vitro in grossen Dosen hemmend, aber in kleinen erhöhend zu wirken, weiter in der dritten Mitteilung, dass Atropin in kleineren Dosen sie hemmt, wïhrend es in grösseren, wenn auch nicht ganz deutlich, sie doch mchr fördert. Nun ergabsich bei der Bobbachtung des Blutatmungszustandes uach gleichzeitigem Zusatz von Atropin und Adrenalin zum Blut in vitro Folgendes (Figg. 4, 5 u. Tabb. 6, 7, 8).

Tab. 6 zeigt den Sanerstoffverbrauch beim Zusatz einer grösseren Menge Adrenalin-Atropingemisch (0,075 my Atropin, 0,075 mg Adrenalin) zu $1 \mathrm{~cm}$ Blut. Diese Tabelle zeigt, dass der Sauerstoffkonsum des Adrenalin-Atropinbluts in allen Fïllen gegenüber dem der Kontrolle keinen grossen Untersehied aufweist (Fig. 4). Setzt man je für sich allein von 


\section{Tabelle 6.}

Der Einfluss von Adrenalin + Atropin auf den Sauerstoffverbrauch des Blutes.

(1 ccm Blut : 0,075 $\mathrm{mg}$ Adrenalin $+0,075 \mathrm{mg}$ Atropin)

Ad. At. $=$ Blut mit Adrenalin + Atropin. K.=Kontrolle.

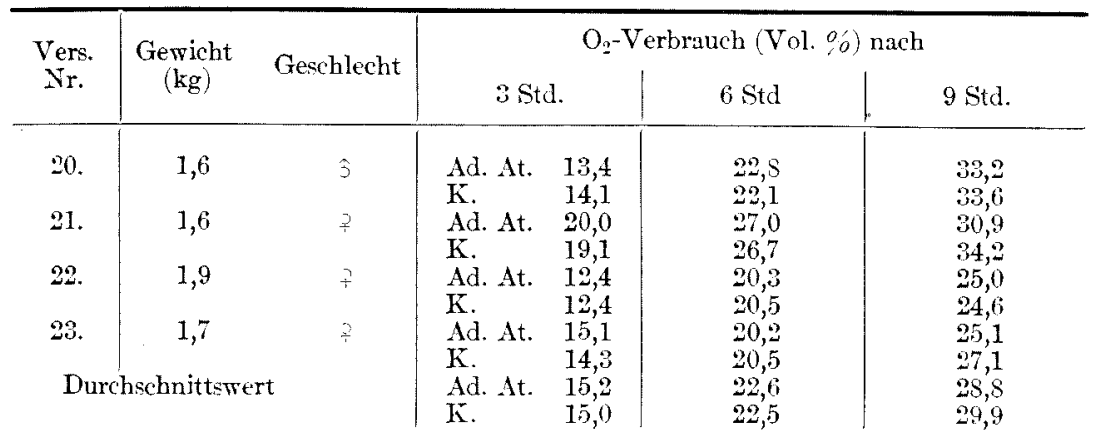

Atropin und Adrenalin dieselbe Dose dem Blut hinzu, so wird auf den Gaswechsel des Bluts von letaterem eine herabsetzende und von ersterem eine mehr fördernde Wirkung ausgeübt, aber bei gleichzeitigem Zusatz beider, wie aus obiger Tabelle ersichtlich, kaum ein Einfluss bemerkt, was wohl darauf beruht, dass AdreFig. 4.

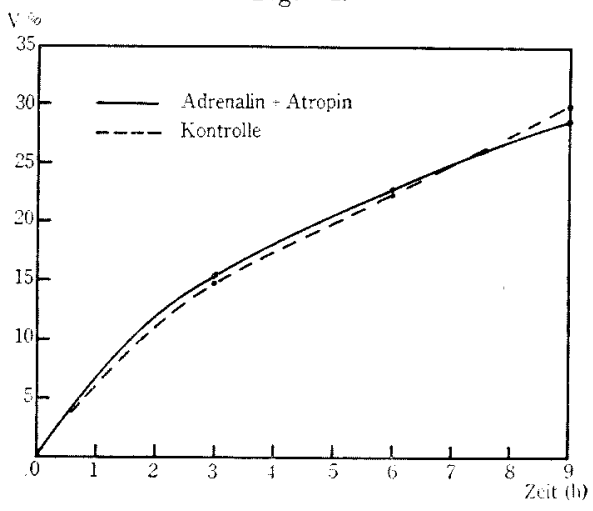
nalin in diesem Falle durch seine hemmende Wirkung den Effekt des Atropins beseitigt hat.

Tab. 7 (Fig. 5) (0,03 Adrenalin u. 0,03 mg Atropin zu $1 \mathrm{ccm}$ Blut) und Tab. 8 (0,005 Adrenalin u. 0,005 $\mathrm{mg}$ Atropin zu $1 \mathrm{ccm}$ Blut) veranschaulichen den Sauerstoffverbrauch des Bluts bei Zusatz einer kleineren Dose beider Gifte zum Blut. Jetzt zeigt sich ein ganz anderes Verhalten gegenüber dem Versuch mit grösseren Dosen. Das mit beiden Giften versehene Blut verbraucht offenbar eine kleinere Menge Sauerstoff als das der Kontrolle.

Eigentlich ergeben die Sympathikusreizung und Parasympathikuslähmung denselben Effekt, so dass man vermuten kann, dass bei gleichzeitiger Anwendung eines sympathikusreizenden und parasympathikuslihmenden 


\section{Tabelle 7.}

Der Einfluss von Adrenalin + Atropin auf den Sauerstoffverbrauch des Blutes.

(1 ccm Blut : 0,03 mg Adrenalin $+0,03 \mathrm{mg}$ Atropin)

Ad. $A t .=$ Blut mit Adrenalin + Atropin. $K .=$ Kontrolle.

\begin{tabular}{|c|c|c|c|c|c|c|}
\hline \multirow{2}{*}{$\begin{array}{l}\text { Vers. } \\
\text { Nr. }\end{array}$} & \multirow{2}{*}{$\begin{array}{c}\text { Gewicht } \\
\text { (kg) }\end{array}$} & \multirow{2}{*}{ Geschlecht } & \multicolumn{4}{|c|}{$\mathrm{O}_{2}$-Verbrauch (Vol. 0 ) nach } \\
\hline & & & $8 \mathrm{Std}$. & & 6 Std. & $9 \mathrm{Std}$. \\
\hline \multirow[t]{2}{*}{24.} & 1,7 & $s$ & Ad. At. & 23,5 & 30,0 & 86,0 \\
\hline & & & $\mathrm{K}$ & 26,0 & 32,5 & 39,0 \\
\hline 25. & 1,5 & f & $\begin{array}{l}\text { Ad. At. } \\
\text { K. }\end{array}$ & $\begin{array}{r}10,0 \\
97\end{array}$ & 12,5 & $\begin{array}{l}15,9 \\
200\end{array}$ \\
\hline \multirow[t]{2}{*}{26.} & 1,8 & $c^{2}$ & Ad. At. & 13,1 & 17,5 & 20,6 \\
\hline & & & $\mathrm{K}$. & 13,1 & 20,2 & 22,2 \\
\hline 27. & 1,8 & $千$ & Ad. At. & 9,3 & 15,3 & 22,7 \\
\hline \multirow{2}{*}{\multicolumn{3}{|c|}{ Durchschnittswert }} & K. & $\begin{array}{l}11,7 \\
140\end{array}$ & $\begin{array}{l}19,0 \\
188\end{array}$ & $\begin{array}{l}24,0 \\
238\end{array}$ \\
\hline & & & $\mathrm{K}$. & 15,1 & 21,5 & 26,3 \\
\hline
\end{tabular}

Giftes die sympathikusreizende oder parasympathikuslähmende Wirkung verdoppelt wird. Dann ist es denkbar, dass bei gleichzeitigem Zusatz von Adrenalin und Atropin die Wirkung des einen von ihnen in doppelter Stärke in die Erscheinung tritt. Dass jcdoch bei gleichzeitigem '/usatz beider Gifte in relativ

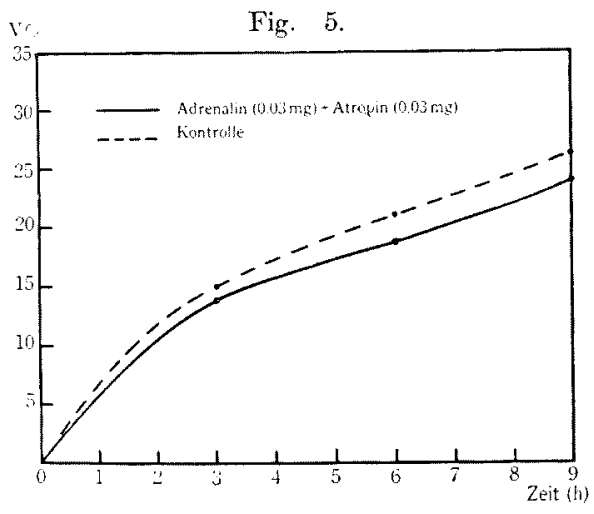

T'abelle 8.

Der Einfluss von Adrenalin $+\Lambda$ tropin auf den Sauerstofverbratuch des Blutes.

(1 com Blut : 0,005 mg Adrenalin $+0,005 \mathrm{mg}$ Atropin)

Ad. At. = Blut mit Adrenalin + Atropin. K. $=$ Kontrolle.

\begin{tabular}{|c|c|c|c|c|c|c|}
\hline \multirow{2}{*}{$\begin{array}{l}\text { Vers. } \\
\text { Nr. }\end{array}$} & \multirow{2}{*}{$\begin{array}{l}\text { Gewicht } \\
\text { (kg) }\end{array}$} & \multirow{2}{*}{ Geschlecht } & \multicolumn{4}{|c|}{$\mathrm{O}_{2}$-Verbrauch (Vol. $\%$ ) nach } \\
\hline & & & \multicolumn{2}{|c|}{$3 \mathrm{Std}}$. & $6 \mathrm{Std}$ & 9 Std. \\
\hline 28 & 1,9 & 3 & $\Lambda \mathrm{d}$. At. & $\begin{array}{l}16,2 \\
16,6\end{array}$ & 18,6 & 28,3 \\
\hline \multirow[t]{2}{*}{29} & 1,6 & Q & Ad. At. & 34,2 & 37,3 & 40,8 \\
\hline & & & & 35,5 & 39,4 & 42,9 \\
\hline 30. & 2,0 & i. & $\underset{\mathrm{K}}{\text { Ad. At. }}$ & $\begin{array}{l}18,2 \\
202\end{array}$ & $\begin{array}{l}22,3 \\
25,1\end{array}$ & $\begin{array}{l}28,6 \\
30,9\end{array}$ \\
\hline 31. & 1,5 & 9 & $\begin{array}{l}\text { Ad. At. } \\
\mathrm{K} .\end{array}$ & $\begin{array}{l}13,8 \\
14,5\end{array}$ & $\begin{array}{l}19,8 \\
20,1\end{array}$ & $\begin{array}{l}24,2 \\
25,6\end{array}$ \\
\hline \multicolumn{3}{|c|}{ Durchschnitswert } & $\begin{array}{l}\text { Ad. At. } \\
\text { K. }\end{array}$ & $\begin{array}{l}20,6 \\
21,7\end{array}$ & $\begin{array}{l}24,5 \\
26,7\end{array}$ & $\begin{array}{l}30,5 \\
33,2\end{array}$ \\
\hline
\end{tabular}


kleinen Mengen zu den isolierten Blutzellen die Blutatmung so verändert wird, als ob Atropin fast allein wirkte, rührt wohl daher, dass die Adrenalinwirkung durch Atropin beseitigt wird.

Zusammenfassung: Beigleichzeitigem Zusatz relativ grosser Dosen von Adrenalin und Atropin zum Blut wird dessen Saucrstoffverbrauch kaum beeinflusst, aber bei relativ kleinen Dosen auffallend herabgesetzt.

\section{Kombinierte Wirkung von Adrenalin und Pilokarpin.}

Selbstverständlich entfaltet Adrenalin und Pilokarpin jedes für sich auf den Gesamtorganismus eine eigentliche Wirkung, und dass jedes für sich auch noch in eigentümlicher Weise auf die Atmung der isolierten Blutzellen wirkt, das wurde schon besprochen. Jetzt habe ich beide Gifte in verschiedenen Mengen gleichzeitig dem Blut zugefügt und die dadurch zustande konmende Veränderung der Blutzellatmung an Hand des Sauerstoffkonsums des Bluts ermittelt, was folgende Resultate ergab (Tab. 9 u. Fig. 6).

Tabelle 9.

Der Einfluss von Adrenalin + Pilokarpin auf den Sauerstofferbrauch des Elutes. $1 \mathrm{ccm}$ Blut : 0,0005 mg Adrenalin (I), 0,05 mg Adrenalin +0,0005 mg Pilokarpin (II), $0,0005 \mathrm{mg}$ Adrenalin $+0,0005 \mathrm{mg}$ Pilokarpin (III). K. Kontrolle.

\begin{tabular}{|c|c|c|c|c|c|c|}
\hline \multirow{2}{*}{$\begin{array}{l}\text { Vers. } \\
\text { Nr. }\end{array}$} & \multirow{2}{*}{$\begin{array}{c}\text { Gewicht } \\
\text { (kg) }\end{array}$} & \multirow{2}{*}{ Geschlecht } & \multicolumn{4}{|c|}{$\mathrm{O}_{2}$-Verbrauch (Vol. \%) nach } \\
\hline & & & & & $6 \mathrm{Std}$. & $9 \mathrm{Std}$. \\
\hline \multirow[t]{4}{*}{32.} & 1,9 & $\varepsilon$ & I. & 12,2 & 17,2 & 21,7 \\
\hline & & & II. & 11,8 & 16,8 & 20,5 \\
\hline & & & III. & 11,9 & 16,6 & 19,9 \\
\hline & & & K. & 11,6 & 16,8 & 20,3 \\
\hline \multirow[t]{4}{*}{33.} & 1,7 & 5 & I. & 10,6 & 16,2 & 18,9 \\
\hline & & 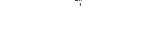 & II. & 10,6 & 15,4 & 18,8 \\
\hline & & & III. & 10,3 & 15,1 & 18,3 \\
\hline & & & $\mathrm{K}$ & 10,6 & 15,2 & 18,5 \\
\hline \multirow[t]{4}{*}{34.} & 1,8 & $\dot{\partial}$ & I. & 9,2 & 16,4 & 21,9 \\
\hline & 1,0 & 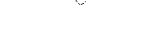 & II. & 8,9 & 16,0 & 21,6 \\
\hline & & & III. & 8,3 & 15,8 & 20,7 \\
\hline & & & $\mathrm{K}$. & 8,5 & 15,8 & 21,3 \\
\hline \multirow{4}{*}{35.} & 18 & f & I. & 13,5 & 18,8 & 23,5 \\
\hline & & & II. & 13,2 & 17,9 & 22,9 \\
\hline & & & III. & 13,0 & 17,8 & 22,6 \\
\hline & & & $\mathrm{K}$ & 13,2 & 18,0 & 22,7 \\
\hline \multirow{4}{*}{86.} & 1,9 & 8 & I. & 15,2 & 21,0 & 27,5 \\
\hline & & & II. & 14,9 & 20,0 & 26,9 \\
\hline & & & III. & 14,6 & 19,8 & 26,8 \\
\hline & & & K. & 14,7 & 20,1 & 26,9 \\
\hline \multirow{4}{*}{\multicolumn{3}{|c|}{ Durchschnittswert }} & I. & 12,1 & 17,9 & 22,9 \\
\hline & & & II. & 11,9 & 17,2 & 22,1 \\
\hline & & & III. & 11,6 & 17,0 & 21,7 \\
\hline & & & $\mathrm{K}$ & 11,7 & 17,2 & 21,9 \\
\hline
\end{tabular}


Fig. 6.

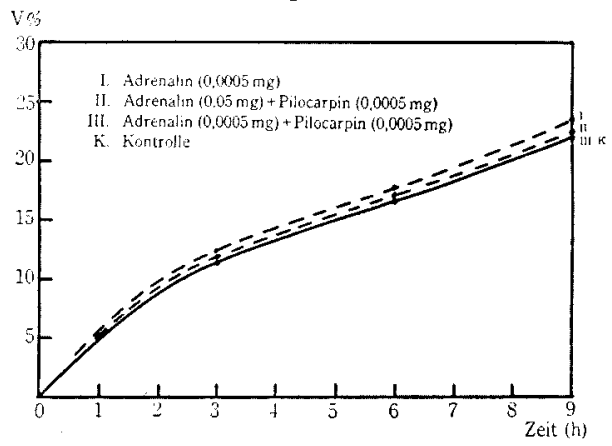

In der Tabelle zeigt I den Sauerstoffverbrauch des Bluts beim Zusatz einer kleinen Dose Adrenalin allein (0,0005 mg Adrenalin : 1 ccm Blut) zum Blut, wo er in allen Fällen gefördert zu sein scheint, II einen solchen bei gleichzeitigem Zusatz einer relativ grossen Menge $(0,05 \mathrm{mg})$ Adrenalin und einer relativ

kleinen Menge $(0,0005 \mathrm{mg})$ Pilokarpin zu $1 \mathrm{ccm}$ Blut, wobei die Blutzellatmung von der des Kontrollbluts nicht wesentlich abweicht, und III einen solchen bei gleichzeitigem Zusatz von Adrenalin und Pilokarpin in relativ kleiner Dose zum Blut (je $0,0005 \mathrm{mg}: 1 \mathrm{ccm}$ Blut), in welchem Falle der Verlauf der Blutatmung fast gleich ist wie bei II. Dass bei Zusatz cines Gemisches aus $0,05 \mathrm{mg}$ Adrenalin und 0,0005 mg Pilokarpin die Sauerstoffatmung des Bluts sich nicht merklich verändert, kommt wohl daher, dass die Wirkung des Pilokarpins, sie zu erhöhen, durch das herabsetzende Vermögen des Adrenalins in relativ grosser Dose vernichtet wird. Doch müsste, da Adrenalin in relativ kleiner Dose, wie I zeigt, die Blutzellatmung deutlich erhöht und Pilokarpin die gleiche Tendenz hat, bei gleichzeitigem Zusatz beider Gifte in relativ kleinen Dosen die Atmung des Bluts in vitro deutlich verstärkt werden. Aber dass dies in Wirklichkeit nicht der Fall ist, könnte auf den ersten Blick paradox erscheinen. Dies lässt sich wohl durch die Annahme erklären, dass vielleicht das Zusammenwirken beider Gifte, jedes in relativ kleiner Menge, cinen Effekt hervorbringt, als ob eines von ihnen allein in relativ grosser Dose cingewirkt hätte.

Zusammenfassung: Bei gleichzeitigem Zusatz von Adrenalin und Pilokarpin zum Blut in vitro lässt sich seine Sauerstoffutmung nicht wesentlich verändern.

5. Kombinierte Wirkung von Adrenalin und Insulin.

Durch verschiedene experimentelle und klinische Beobuchtungen ist bestätigt worden, dass Nebenniere und Pankreas auf den Gesamtorganis- 
mus und die isolierten Organe durch ihre Inkrete einwirken und zwar die erstere sympathikusreizend und das letztere parasympathikuserregend, also antagonistisch zueinander.

Zuel ze r ${ }^{4)}$ Frugon i5) und Comessatti $\left.{ }^{6}\right)$ u. a. berichten, dass der Pankreassaft die Fähigkeit hat, die Adrenalinglykosurie zu verhindern, wenn er in grosser Menge vor dem Adrenalin injiziert wird. Loew i 7 hat festgestellt, dass Pankreas die Funktion besitzt, sympathische Hemmung zu erregen bzw. die Reizbarkeit sympathischer Förderungsnerven herabzusetzen. Kita mura $\left.\mathrm{a}^{8}\right)$ hat durch Untersuchung an Kaninchen bestïtigt, dass durch Injektion von 0,5 ccm 0,1\% iges Adrenalin entstehende Hyperglykïmie und Glykosurie durch $0,2 \mathrm{ccm}$ Insulin ausgeschaltet wird. K ylin, ${ }^{9)} \mathrm{Cse}_{\mathrm{p} a \mathrm{i}}$ und $W$ eiss ${ }^{10)}$ u. a. betonen von kardiovaskulürer Seite her, dass Insulin und Adrenalin aufeinander antagonistisch wirken, und Gottsch al k,11) dass durch Insulin in grossen Dosen bei Fröschen innerhalb drei Stunden Streckkrämpfe erzeugt werden, die durch nachfolgende Adrenalininjektion zurückgehen. Boden und W ankel112) fanden am isolierten Meerschweinchendickdarm Insulin und Adrenalin gegeneinander antagonistisch einwirken. Kogan und Ponirowsk $y^{13}$ ) haben bemerkt, dass Insulin in kleinen, nicht toxischen Dosen nur eine schwache und kurzdauernde Blutdruckherabsetzung hervorruft, jedoch bereits die blutdruckerböhende Wirkung des Adrenalins hemmt; dass bedeutende, toxische Insulindosen den Blutdruck, wenn auch gleichfalls nur auf kurze Zeit, deutlich herabsetzen und die druckerhöhende Wirkung entsprechend den grösseren Adrenalindosen hemmen, und dass eine kleine Menge Insulin keine Salivation hervorruft; ferner, dass bei gleichzeitiger Anwendung von Insulin und Adrenalin die durch Injektion des letzteren auftretende Salivation gehemmt wird, dass eine grosse toxische Insulinmenge nicht nur starke Speichelabsonderung, sondern auch allgemeine Reaktion hervorruft, welche aber durch entsprechend grosse Adrenalindose sofort neutralisiert und dadurch verhindert wird.

Zu der Untersuchung, wie der Gaswechsel der Blutzellen durch Zusammenwirken beider Gifte beeinflusst wird, habe ich den Sauerstoffverbrauch des Bluts in vitro, welches mit ihnen versetzt wurde, gemessen. Tab. 10 stellt die Sauerstoffzehrung bei gleichzeitigem Zusatz beider Gifte in grösserer Dose $(0,075 \mathrm{mg}$ Adrenalin und 0,075 ccm Insulin-Tronto: 1 con Blut) dar.

Aus obiger Tabelle ist ersichtlich, dass der Sauerstoffkonsum des Bluts bei Zusatz der Giftmischung meistens mehr oder weniger getördert wird gegenüber dem des Kontrollbluts. Es war öfters davon die Rede, dass der Sauerstoffverbrauch des Bluts durch eine grosse Menge Adrenalin deutlich

4) Zuelzer, Berl. kl. Wochenschr., 1901, 1209.

5) Frugoni, Berl. kl. Wochenschr., 1908, 1606.

6) Co messatti, Arch. f. exp. Path. u. Pharm., 1909, 60, 243.

7) Loewi, Münch. med. Wochenschr., 1907, 1411.

8) Kit a mu r a, Nippon-Naika-Gakkai-Zasshi, 1924-25, 12, 1068 (jap.).

9) K y lin, Med. Kl., 1925, 1262; Skand. Arch. f. Physiol., 1925, 46, 321.

10) Csépa $i$ und Weiss, Wien. Arch. f. inn. Med., 1925, 10, 195.

11) Gottschalk, Biochem. Zeitschr., 1925, 159, 502.

12) Boden und Wankell, Kl. Wochenschr., 1925, 1828.

13) Kogan und Ponirow sky, Zeitschr. f. d. ges. exp. Med., 1925, 47, 557. 


\section{Tabelle 10.}

Der Finfluss ron Adrenalin +Insulin auf den Sauerstofferbrauch des Blutes. (1 cem Blut : $0,075 \mathrm{mg}$ Adrenalin $+0,075 \mathrm{ccm}$ Insulin) In. Ad. $=$ Blut mit Insulin + Adrenalin. $\quad \mathrm{K} .=$ Kontrolle.

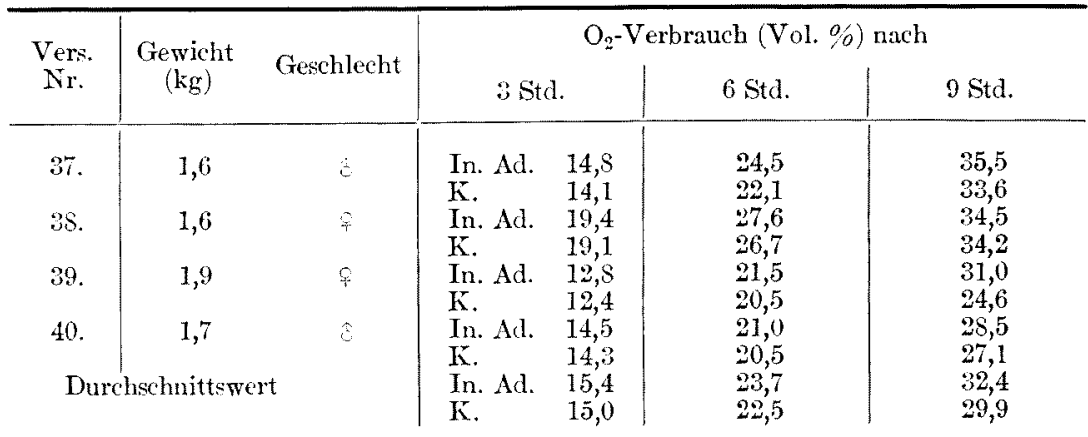

herabgesetzt, aber durch eine grosse Dose Insulin allein meist gesteigert wird. Und wenn man, wie die obige Tabelle zeigt, ein Gemisch aus beiden Giften dem Blut zusetzt, so lässt sich sein Sauerstoffverbrauch, wenn auch nicht erheblich, fördern, was wohl daher rührt, dass die fördernde Wirkung des Insulins die hemmende Kr raft des Adrenalins überwindet.

Bei gleichzeitigem Zusatz cines Gemisches aus Adrenalin und Insulin im Verhältnis von $0,005 \mathrm{mg}$ resp. $0,03 \mathrm{mg}$ Adrenalin und $0,005 \mathrm{~cm}$ resp. $0,003 \mathrm{ccm}$ Insulin : $1 \mathrm{~cm}$ Blut crgibt sich das in Tabb. 11 (Fig. 7 ) resp. 12 angegebene Resultat. Wie auf den ersten Blick ersichtlich, ist in den beiden Versuchsreihen der Sanerstoffverbrauch des mit Giftmischung ver-

\section{Tabelle 11.}

Der Finfluss von Adrenalin + Insulin auf den Sauerstoffverbrauch des Blutes.

(1 ccm Blut : 0,08 $\mathrm{mg}$ Adrenalin $+0,03 \mathrm{ccm}$ Insulin)

Ad. In.=Blut mit Adrenalin +Insulin. K. $=$ Kontrolle.

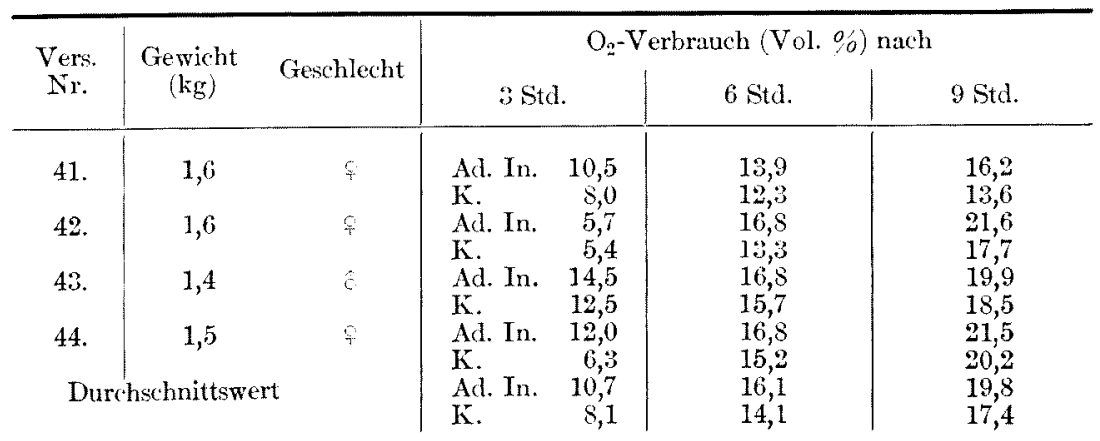




\section{Tabelle 12.}

Der Einfluss ron Adrenalin + Insulin auf den Sauerstoffrerbrauch des Blutes.

( $1 \mathrm{~cm}$ Blut : 0,005 $\mathrm{mg}$ Adrenalin +0,005 cm Insulin)

Ad In.=Blut mit Adrenalin + Insulin. K.=Kontrolle.

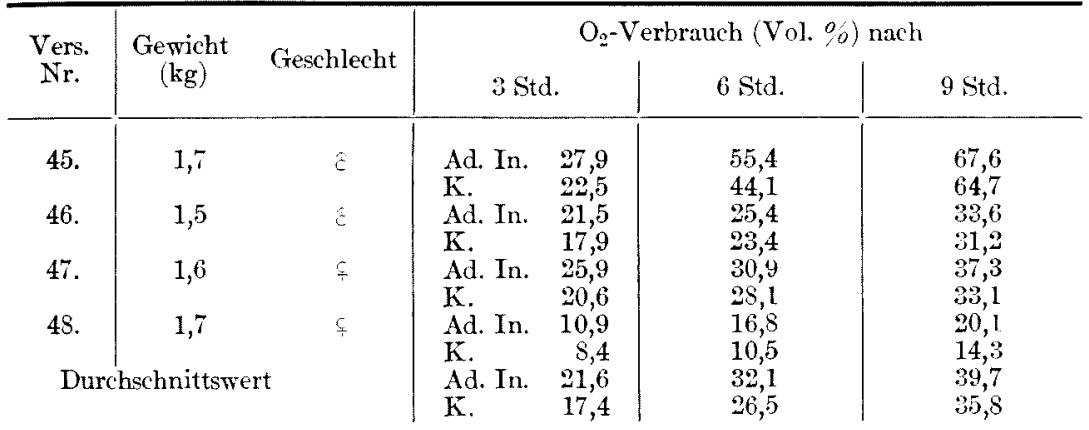

Fig. 7.

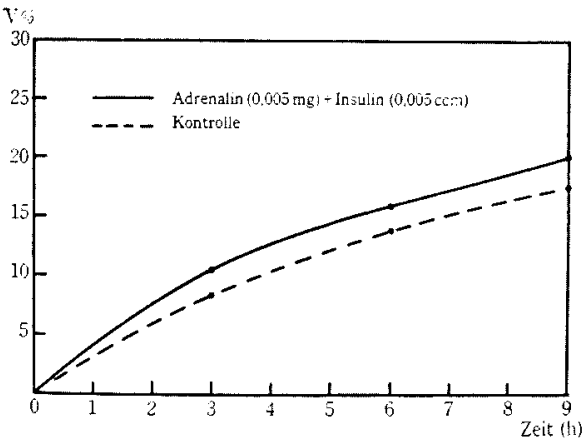

sehenen Bluts gegenüber dem bei der Kontrolle bedeutend erhöht. Es ist schon festgestellt worden, dass, wenn das Blut mit relativ kleiner Dose des einen der beiden Gifte versetzt wird, sein Gaswechsel in allen Fällen sich deutlich fördern lässt. Und bei gleichzeitigem Zusatz beider in kleinerer Dose

resultiert, wie aus obiger 'Tabelle ersichtlich, deutlich erhöhte Atmung des Bluts.

Boden und Wankell12 berichteten, dass sich am Trendelenburgschen Froschschenkelpräparat unter Insulin konstante Abnahme der Tropfenzabl zeigte, dass es also an diesem Präparate im gleichen Sinne zu wirken scheint wie Adrenalin, und A h l g r e $n^{2}$ sagte : „Bei gleichzeitigem Zusatz von Insulin und Adrenalin findet man diese Hormone in einer gewissen Konzentration ihre beschleunigende Wirkung auf die Gewebsatmung gegenseitig völlig aufheben. Bei einer anderen Konzentration erbält man unvollstïndige IIemmung oder Dominanz entweder der Insulin- oder Adrenalinwirkung. Insulin und Adrenalin sind also in ihrer kalorigenen Wirkung gleichzeitig Antagonisten und Agonisten."

Auch meine Untersuchung kann wie die der oben genannten Autoren so aufgefasst werden, dass Adrenalin und Insulin in relativ grossen Dosen antagonistisch, aber in relativ kleinen synergetisch wirken. 
Zusammenfassung: Fügt man Insulin und Adrenalin gleichzeitig dem Blut hinzu, so scheinen sie auf die Sauerstoffatmung des Bluts in relativ grossen Dosen antagonistisch, aber in relativ kleinen im Gegenteil mehr oder weniger synergetisch zu wirken. Der Sauerstoffverbrauch des Bluts im vitro lässt sich durch die Giftmischung in beiden Dosen tördern.

\section{Kombinierte Wirkung von Adrenalin und Ergotamin.}

Bekanntlich ist Adrenalin ein Sympathikusreizgift und Ergotamin sein Antagonist. Dass von beiden jedes für sich auch auf die Blutzellatmung in vitro ganz entgegengesetzt wirkt, wurde in einer früheren Mitteilung besprochen. Ich habe weiter untersucht, auf welche Weise der Gaswechsel des Bluts beeinflusst wird, wenn man beide gleichzeitig dem letzteren in vitro zufügt, um ihre gegenseitige Beziehung zu ermitteln (Tab. 13 u. Fig. 8). Es zeigt I den Fall, wo eine relativ grosse Dose Ergo-

Tabelle 13.

Der Einfluss von Adrenalin + Ergotamin auf den Sauerstoffverbrauch des Blutes. $1 \mathrm{~cm}$ Flut : 0,05 $\mathrm{mg}$ Ergotamin (I), 0,05 mg Ergotamin +0,05 mg Adrenalin (II), $0,0005 \mathrm{mg}$ Ergotamin $+0,0005 \mathrm{mg}$ Adrenalin (III). K.=Kontrolle.

\begin{tabular}{|c|c|c|c|c|c|c|}
\hline \multirow{2}{*}{$\begin{array}{l}\text { Vers. } \\
\text { Nr. }\end{array}$} & \multirow{2}{*}{$\begin{array}{l}\text { Gewicht } \\
(\mathrm{kg})\end{array}$} & \multirow{2}{*}{ Geschlecht } & \multicolumn{4}{|c|}{$\mathrm{O}_{2}-$ Verbrauch (Vol. \%) nach } \\
\hline & & & & & $6 \mathrm{Std}$. & $9 \mathrm{Std}$. \\
\hline \multirow[t]{4}{*}{49.} & 2,0 & $\hat{3}$ & I. & 13,3 & 18,3 & 29,6 \\
\hline & & & II. & 12,3 & 17,4 & 28,2 \\
\hline & & & III. & 12,9 & 17,5 & 25,9 \\
\hline & & & $\mathrm{K}$. & 12,4 & 17,6 & 28,2 \\
\hline \multirow[t]{4}{*}{50} & 2,1 & $\vdots$ & I. & 22,8 & 34,3 & 43,0 \\
\hline & & & II. & 21,6 & $: 33,4$ & 42,2 \\
\hline & & & III. & 31,9 & 33,6 & 42,1 \\
\hline & & & $\mathrm{K}$ & 22,0 & 33,8 & 41,9 \\
\hline \multirow[t]{4}{*}{51.} & 2,1 & $j$ & I. & 18,2 & 31,3 & 44,2 \\
\hline & & & II. & 16,4 & 29,9 & 42,7 \\
\hline & & & III. & 16,9 & 28,7 & 42,5 \\
\hline & & & $\mathrm{K}$. & 16,1 & 30,1 & 42,9 \\
\hline \multirow{4}{*}{$5:$} & 1,8 & 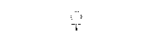 & I. & 129 & 20,5 & 29,8 \\
\hline & & & II. & 10,8 & 18,9 & 27,9 \\
\hline & & & III. & 11,0 & 18,8 & 28,1 \\
\hline & & & $\mathrm{K}$. & 10,9 & 18,6 & 27,8 \\
\hline \multirow{4}{*}{53.} & 1,7 & 3 & I. & 13,1 & 22,4 & 30,3 \\
\hline & & & II. & 12,5 & 21,8 & 28,9 \\
\hline & & & III. & 12,3 & 21,5 & 28,8 \\
\hline & & & $\mathrm{K}$. & 12,6 & 21,6 & 29,0 \\
\hline \multirow{4}{*}{\multicolumn{3}{|c|}{ Durchechnittswert }} & I. & 16,1 & 25,4 & 35,4 \\
\hline & & & II. & 14,7 & 24,3 & 34,0 \\
\hline & & & $11 \mathrm{I}$. & 15,0 & 24,0 & 34,1 \\
\hline & & & K. & 14,8 & 24,3 & 34,0 \\
\hline
\end{tabular}


Fig. 8.

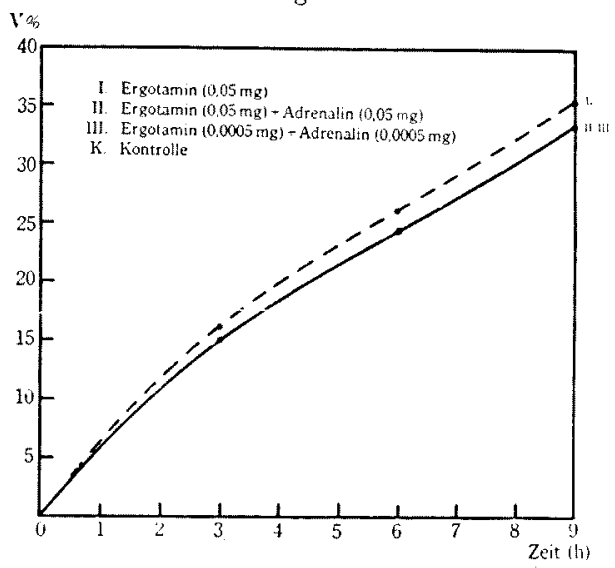

taminum tartaricum $(0,05$ $\mathrm{mg}$ Ergotamin : 1 ccm Blut) allein dem Blut zugesetzt wurde und wo, wie auf den ersten Blick ersichtlich, die Blutzellatmung gesteigert wird, II einen solchen mit gleichzeitigem $\mathrm{Zu}-$ satz von Adrenalin und Ergotamin in relativ grossen Dosen (je 0,05 mg zu $1 \mathrm{ccm}$ Blut), wo alle Versuche fast gleich ausfielen wie die Kontrolle, und III einen

solchen mit relativ kleinen Dosen (je $0,0005 \mathrm{mg}$ ) beider Gifte, wo sich auch wie bei II ein ähnliches Ergebnis wie bei der Kontrolle zeigte. Also wird bei II und III die Blutatmung weder verstärkt noch gehemmt. Nach der Beobachtung von Ahlgren ${ }^{2}$ zeigten bez. des Stoffwechsels der Froschmuskeln das gleichzeitig hinzugefügte Adrenalin und Ergotamin Tendenz zu gegenseitig hemmender Wirkung. Auch meine Versuche lassen erkennen, dass die hemmende Phase des Adrenalins resp. Ergotamins durch die erhöhende Wirkung des letzteren resp. ersteren aufgehoben wird, dass also beide gegenseitig ihre Wirkung geltend machen. So kann man sagen, dass Adrenalin und Ergotamin nicht nur auf den Gesamtorganismus oder die einzelnen Organe, sondern auch auf den Gaswechsel der isolierten überlebenden Erythrozyten in entgegengesetztem Sinne wirken.

Zusammenfassung: Adrenalin und Ergotamin wirken, dem Blut gleichzeitig zugesetzt, auf seine Sauerstoffzehrung weder verstärkend noch hemmend. Also scheinen beide auf die Blutzellatmung in vitro in entgegengesetztem Sinne zu wirken.

7. Kombinierte Wirkung von Eryotamin und Atropin.

Es wurde schon mehrmals erwähnt, dass Ergotamin in grossen Dosen die Blutzellatmung erhöht, aber in kleinen dazu neigt, sic herabzusctzen, und auch Atropin in kleinen auf sie hemmend wirkt, während ciner grösseren Menge mchr oder minder fördernde Wirkung zukomnt. Um den Einfluss des gleichzeitigen Zusatz es beider Gifte auf den Simerstoffver- 
brauch des Bluts in vitro zu beobachten, habe ich Versuche angestellt, deren Ergebnisse in Tab. 14 wiedergegeben sind.

\section{Tabelle 14.}

Der Einfluss von Atropin + Ergotamin auf den' Sauerstoffverbrauch des Blutes.

$1 \mathrm{~cm}$ Blut : 0,0005 $\mathrm{mg}$ Atropin $+0,05 \mathrm{mg}$ Ergotamin (I), 0,0005 mg Atropin + 0,0005 mg Ergotamin (II), 0,0005 $\mathrm{mg}$ Atropin (III). K. = Kontrolle.

\begin{tabular}{|c|c|c|c|c|c|c|}
\hline \multirow{2}{*}{$\begin{array}{l}\text { Vers. } \\
\text { Nr. }\end{array}$} & \multirow{2}{*}{$\begin{array}{l}\text { Gewicht } \\
\text { (kg) }\end{array}$} & \multirow{2}{*}{ Geschlecht } & \multicolumn{4}{|c|}{$\mathrm{O}_{2}$-Verbrauch (Vol. \%) nach } \\
\hline & & & \multicolumn{2}{|c|}{$3 \mathrm{Std}$} & $6 \mathrm{Std}$. & $9 \mathrm{Std}$ \\
\hline \multirow[t]{4}{*}{54.} & 1,9 & j & I. & 10,9 & 16,7 & 22,1 \\
\hline & & & II. & 10,5 & 15,2 & 21,9 \\
\hline & & & III. & 10,7 & 16,6 & 22,1 \\
\hline & & & $\mathrm{K}$ & 11,5 & 16.9 & 22,6 \\
\hline \multirow[t]{4}{*}{55.} & 1,9 & $\therefore$ & I. & 12,6 & 20,2 & 25,5 \\
\hline & & & II. & 11,9 & 20,1 & 24,0 \\
\hline & & & III. & 12,6 & 20,8 & 24,0 \\
\hline & & & K. & 12,8 & 20,9 & 25,9 \\
\hline \multirow[t]{4}{*}{56 . } & 1,5 & 3 & I. & 12,8 & 20,2 & 29,0 \\
\hline & & & II. & 12,2 & 19,2 & 27,7 \\
\hline & & & III. & 12,6 & 19,7 & 28,9 \\
\hline & & & $\mathrm{K}$ & 13,0 & 20,9 & 29,5 \\
\hline \multirow[t]{4}{*}{57.} & 1,8 & $\ddot{\vec{r}}$ & I. & 10,5 & 14,0 & 17,5 \\
\hline & & & II. & 10,0 & 13,8 & 17,0 \\
\hline & & & III. & 10,4 & 13,8 & 17,3 \\
\hline & & & $\mathrm{K}$. & 10,8 & 14,5 & 17,6 \\
\hline \multirow[t]{4}{*}{58.} & 1,9 & 3 & I. & 14,0 & 21,5 & 28,6 \\
\hline & & & II. & 13,9 & 21,2 & 27,8 \\
\hline & & & III. & 14,0 & 21,1 & 28,0 \\
\hline & & & $\mathrm{K}$. & 14,8 & 22,1 & 28,7 \\
\hline \multirow{4}{*}{\multicolumn{3}{|c|}{ Durchschnittstrert }} & I. & 12,2 & 18,5 & 24,5 \\
\hline & & & II. & 11,7 & 17,9 & 23,7 \\
\hline & & & III. & 12,1 & 184 & 24,1 \\
\hline & & & $\mathrm{K}$. & 12,6 & 19,1 & 24,9 \\
\hline
\end{tabular}

Fig. 9 .

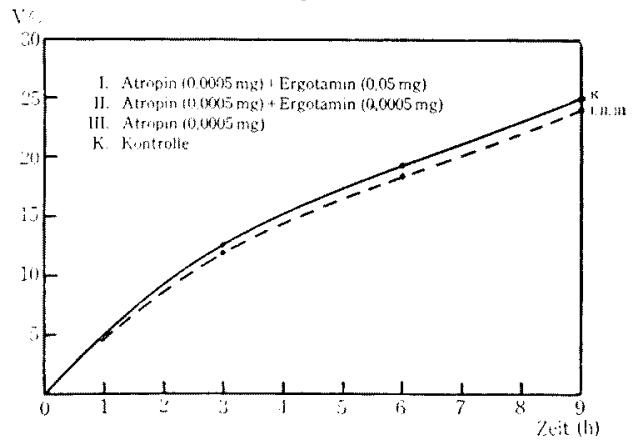

In Tab. 14 (Fig. 9) zeigt I die Sauerstoffzehrung des Bluts bei gleichzeitigem Zusatz von Ergotamin und Atropin im Verhältnis von resp. 0,05 und $0,0005 \mathrm{mg}$ zu $1 \mathrm{ccm}$. Hier ist sie ein wenig kleiner als bei der Kontrolle. II zeigt den Sanerstoffverbrauch der Blutzellen bei gleichzeitigem Einwirkenlassen von Atropin und Ergotamin auf das Blut, und zwar im Verhültnis von resp. $0,0005 \mathrm{mg}$ und $0,00005 \mathrm{zu} 1 \mathrm{ccm}$. 
Hiernach verhält er sich ebenso wie bei I, und dic Blutzellatmung neigt deutlich zur Hemmung. Bei III handelt es sich um Experimente, in denen Atropin allein in kleiner Dose $(0,0005 \mathrm{mg})$ zugesetzt wurde. Auch hier zeigt sich Tendenz zur Hemmung.

Bei der Zusammenfassung der Ergebnisse dieser drei Versuchsreihen ergibt sich, dass bei gleichzeitigem Einwirkenlassen von Ergotamin und Atropin immer nur Atropin seine Wirkung geltend macht, ohne dass das Ergotamin selbst zur. Wirkung kommt, also dass die Wirkung des letzteren von der des ersteren anfgehoben wird. Es geht daraus hervor, dass Atropin, welchem bekanntlich das Vermögen zukommt, die Wirkung der Parasympathikusreizgifte aufzuheben, auch den Ergotamineffekt auf den Sauerstoffverbrauch des Bluts in vitro, sei er ein fördernder, sei er ein hemmender, beseitigen kann.

Zusammenfassung: Bei gleichzeitigem Einwirkenlassen von Atropin und Ergotamin auf das Blut in vitro macht sich immer nur die Wirkung des ersteren geltend, ohne dass die des letzteren auftritt.

\section{Kombinierte Wirkung von Ergotamin und Pilokarpin.}

Von der alleinigen Wirkung von Ergotamin oder Pilokarpin auf die Blutzellatmung in vitro war schon die Rede. In diesem Kapitel wird auf die Veränderungen der Blutatmung bei gleichzeitigem Zusatz beider Gifte zur Erkenntnis ihrer gegenseitigen Beziehungen eingegangen. In Tab. 15 und Fig. 10 ist das erhaltene Ergebnis wiedergegeben. I zeigt die Grösse der Blutatmung beim Zusatz von Ergotamin und Pilokarpin zum Blut im Verhältnis von resp. 0,05 $\mathrm{mg}$ und 0,0005 $\mathrm{mg}$ zu $1 \mathrm{ccm}$ Blut, II eine solche beim Zusatz beider Gifte in der Dose von je $0,0005 \mathrm{mg}$ und III die Versuchsreihe, wo 0,0005 mg Pilokarpin allein $1 \mathrm{~cm}$ Blut hinzugefügt worden ist.

Aus I ist ersichtlich, dass der Sauerstoffverbrauch in allen Fällen, wenn auch nicht erheblich, so doch im grossen und ganzen dazn neigt, gefördert zu werden; bei II und III ist die Erhöhung des Sauerstoffkonsums ziemlich ausgesprochen.

Pilokarpin wirkt auf die Blutzcllatmung eigentlich nur in relativ kleinen Dosen erhöhend, Ergotamin in relativ grossen nur sch wach steigernd, aber in relativ kleinen Mengen hat es im Gegenteil die Tendenz, sie mehr oder minder zu hemmen. Doch scheint nach dem obigen Resultat bei gleichzoitigem Einwirkenlassen von Ergotamin und Pilokarpin die Wirkung des 
Tabelle 15.

Der Einfluss von Pilokarpin + Ergotamin auf den Sauerstoffverbrauch des Blutes. $1 \mathrm{~cm}$ Blut : 0,05 mg Ergotımin +0,0005 mg Pilokarpin (I), 0,0005 mg Ergotamin + 0,0005 mg Pilokarpin (II), 0,0005 mg Pilokarpin (III). K.=Kontrolle.

\begin{tabular}{|c|c|c|c|c|c|c|}
\hline \multirow{2}{*}{$\begin{array}{l}\text { Vers. } \\
\text { Nr. }\end{array}$} & \multirow{2}{*}{$\begin{array}{c}\text { Gewicht } \\
\text { (kg) }\end{array}$} & \multirow{2}{*}{ Geschlecht } & \multicolumn{4}{|c|}{$\mathrm{O}_{2}$-Verbrauch (Vol. \%) nach } \\
\hline & & & & & $6 \mathrm{Std}$ & $9 \mathrm{Std}$ \\
\hline \multirow[t]{4}{*}{59.} & 1,7 & $i$ & I. & 13,2 & 16,2 & 18,9 \\
\hline & & & II. & 13,9 & 16,6 & 19,5 \\
\hline & & & III. & 13,8 & 16,6 & 19,3 \\
\hline & & & $\mathbf{K}$ & 13,0 & 16,1 & 18,5 \\
\hline \multirow[t]{4}{*}{60.} & 1,6 & 5 & I. & 14,1 & 16,8 & 20,0 \\
\hline & & & II. & 14,3 & 17,9 & 23,2 \\
\hline & & & III. & 14,3 & 17,7 & 23,0 \\
\hline & & & K. & 13,7 & 16,5 & 19,8 \\
\hline \multirow[t]{4}{*}{61.} & 2,0 & $\varsigma$ & I. & 11,4 & 15,7 & 18,3 \\
\hline & & & II. & 12,3 & 16,6 & 19,4 \\
\hline & & & III. & 12,2 & 16,6 & 19,2 \\
\hline & & & $\mathrm{K}$. & 10,6 & 15,4 & 17,1 \\
\hline \multirow[t]{4}{*}{62.} & 1,6 & 6 & I. & 10,7 & 14,7 & 20,0 \\
\hline & & & III. & 11,6 & 15,7 & 25,7 \\
\hline & & & III. & 11,6 & 15,3 & 25,3 \\
\hline & & & K. & 9,9 & 14,1 & 19,5 \\
\hline \multirow[t]{4}{*}{63.} & 1,8 & $\hat{c}$ & I. & 18,9 & 23,5 & 28,6 \\
\hline & & & II. & 19,3 & 24,3 & 30,3 \\
\hline & & & III. & 19,3 & 24,2 & 30,0 \\
\hline & & & K. & 17,7 & 23,0 & 27,7 \\
\hline \multirow{4}{*}{\multicolumn{3}{|c|}{ Durchschnittswert }} & I. & 13,7 & 17,4 & $2 \mathrm{I}, 2$ \\
\hline & & & II. & 14,3 & 18,2 & 23,6 \\
\hline & & & III. & 14,2 & 18,1 & 23,4 \\
\hline & & & $\hat{\mathrm{K}}$. & 13,0 & 17,0 & 20,5 \\
\hline
\end{tabular}

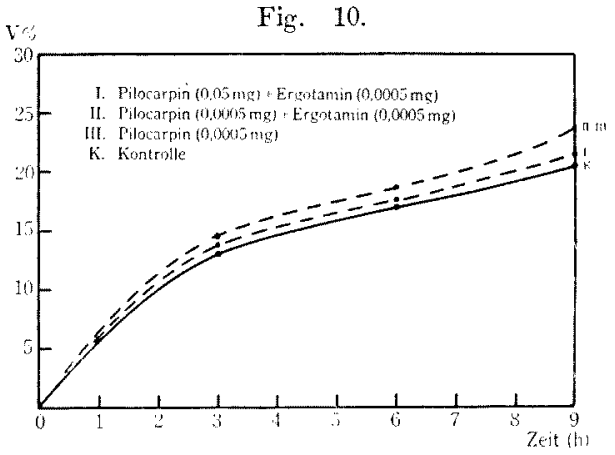

letzteren durch eine relativ grosse Dose des ersteren undeutlich zu werden, während bei Gegenwart einer relativ kleinen Dose Ergotamin nur die Pilokarpinwirkung zum Vorschein kommt. Also hebt Ergotamin in grossen Dosen die Wirkung des Pilokarpins auf die Blutzellatmung in vitro auf und zcigt in kleinen Dosen die Tendenz, im Gegenteil von der Pilokarpinwirkung vernichtet zu werden.

Zusammenfassung: Bei gleichzeitigem Einwirkenlassen von Ergotamin und Pilokarpin auf das Blut in vitro beseitigt ersteres in relativ grossen Menge dic Wirkung des letzteren und erhöht dadurch nur schwach 
die Blutzellatmung, während die hemmende Wirkung von Ergotamin in relativ kleiner Menge durch die Pilokarpinwirkung aufgehoben zu werden scheint und der Sauerstoffkonsum des Bluts erheblich betördert wird.

\section{Kombinierte Wirkung von Pituglandol und Thyreoglandol.}

Dass die Hypophyse und Schilddrüse nicht nur im Gesamtorganismus, sondern auch im Energieumsatz der isolierten ïberlebenden Organe uud Gewebe eine grosse Rolle spielt, wurde von vielen Autoren sowohl klinisch als auch experimentell nachgewiesen, was ich in meiner früheren Arbeit ${ }^{1+\text { ? }}$ besprochen habe. Ich habe in dieser Arbeit bestaitigt, dass Pituglandol (Hypophysenhinterlappenextrakt) auf die Blutzellatmung etwas hemmend, dagegen Thyreoglandol (Schilddrüsenextrakt) deutlich steigernd wirkt. Zur Ermitting davon, anf welche Weise der Gaswechsel des Bluts bei gleichzeitigem Zusatz dieser beiden Inkrete beeinflusst wird, habe ich sie in verschiedenen Mengen zugleich dem Blut in vitro zugefügt und seincn Sauerstoffrerbrauch gemessen.

\section{Tabelle 16.}

Der Einfluss ron Pituglandol $\rightarrow$ Thyrenglandol auf den Sauerstofverbrauch des Blutes.

(1 com Blut : 0,075 con Pituglandol $+0,075 \mathrm{~cm}$ Thyreoglandol)

P. T. = Blut mit Pituglindol + Thyrenglandol. K. $=$ Kontrolle.

\begin{tabular}{|c|c|c|c|c|c|c|}
\hline \multirow{2}{*}{$\begin{array}{l}\text { Vers: } \\
\text { Nr. }\end{array}$} & \multirow{2}{*}{$\begin{array}{c}\text { Gewicht } \\
(\mathrm{kg})\end{array}$} & \multirow{2}{*}{ Geechlecht } & \multicolumn{4}{|c|}{$\mathrm{O}_{2}$-Verbrauch ( Vol. 0.6$)$ nach } \\
\hline & & & \multicolumn{2}{|c|}{$\because$ Sul. } & $6 \mathrm{std}$ & $9 \mathrm{Std}$ \\
\hline \multirow[t]{2}{*}{64.} & 1,6 & 5 & P. $T$. & 15,4 & 19,8 & 21,9 \\
\hline & & & K. & 18,8 & 15,4 & $18, ?$ \\
\hline 65. & 1,5 & ? & $\mathrm{P} . \mathrm{T}$. & 10,4 & $\begin{array}{l}12,3 \\
117\end{array}$ & 17,9 \\
\hline \multirow{2}{*}{66} & 1,6 & 5 & P. T. & 19,4 & 22,4 & 38,9 \\
\hline & & & $\mathrm{K}$. & 19,7 & 21,7 & 35,2 \\
\hline \multirow[t]{2}{*}{67.} & 1,5 & $\therefore$ & $\mathrm{P} . \mathrm{T}$. & 16,9 & 24,2 & 31,1 \\
\hline & & & & $12, ;$ & 22,3 & 25,8 \\
\hline \multirow{2}{*}{\multicolumn{3}{|c|}{ Durchschnitswert }} & P. T. & $15, \tilde{3}$ & 19,7 & 27,5 \\
\hline & & & K. & 13,4 & 17,8 & 24,2 \\
\hline
\end{tabular}

Tab. 16 zeigt den Sauerstoffkonsum des Bluts bei gleichzeitigem Zusatz von Pituglandol (Roche) und Thy reoglandol (Roche) in relativ grosser Menge (0,075 cem Pituglandol und 0,075 ccm Thyreoglandol: $1 \mathrm{~cm}$ Blut); hier ist er in allen Versuchen deutlich gefördert (Fig. 11).

14) Tsukamoto, Tohoku Journ. Exp. MEd., 1929, 12, 179. 


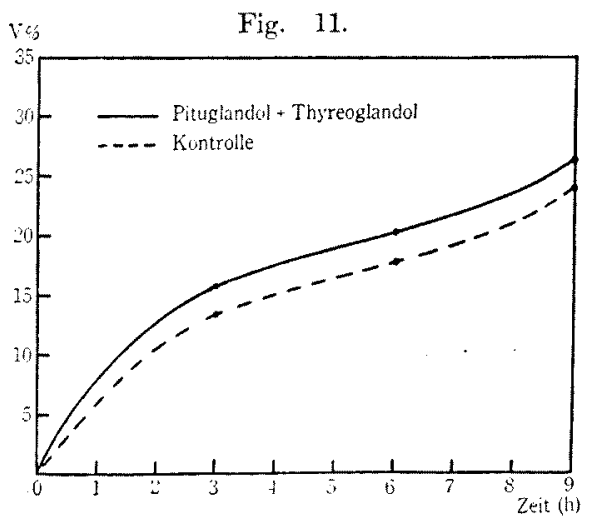

Dann habe ich eine relativ kleine Dose Pituglandol und Thyreoglandol, d. h. die beiden Hormone, dem Blut im Verhältnis von je $0,005 \mathrm{ccm} \mathrm{zu}$ $1 \mathrm{ccm}$ zugesetzt, wobei auch das mit Hormongemisch versetzte Blut einen stärkeren Sauerstoffverbrauch aufwies als das der Kontrolle (Tab. 17).

\section{Tabelle 17.}

Der Finfluss von Pituglandol + Thyreoglandol auf den Sauerstofverbrauch des Blutes.

( 1 cen Blut : $0,005 \mathrm{~cm}$ Pituglandol $+0,005 \mathrm{~cm}$ Thyreoglandol)

P. T. = Blut mit Pituglandol + Thyreoglandol. $\mathrm{K} .=$ Kontrolle.

\begin{tabular}{|c|c|c|c|c|c|c|}
\hline \multirow{2}{*}{$\begin{array}{l}\text { Vers. } \\
\text { Nr. }\end{array}$} & \multirow{2}{*}{$\begin{array}{c}\text { Gewicht } \\
\text { (kg) }\end{array}$} & \multirow{2}{*}{ Geschlecht } & \multicolumn{4}{|c|}{ O. Verbrauch (Vol. \%) nach } \\
\hline & & & & & $6 \mathrm{Std}$ & $9 \mathrm{Std}$. \\
\hline \multirow[t]{2}{*}{68.} & 1,7 & s & P. T. & 13,2 & 14,7 & 16,7 \\
\hline & & & K. & 12,7 & 14,9 & 16,5 \\
\hline 69. & $1, \mathrm{~S}$ & 3 & P. T. & 16,6 & 20,9 & 23,3 \\
\hline \multirow{2}{*}{70} & 1,9 & 8 & $\begin{array}{l}\text { M. } \\
\text { P. T. }\end{array}$ & 16,5 & $\begin{array}{l}16,8 \\
21,2\end{array}$ & $\begin{array}{l}21,8 \\
23,4\end{array}$ \\
\hline & & & K. & 13,2 & 17,3 & 21,8 \\
\hline \multirow[t]{2}{*}{71.} & 2,0 & $?$ & P. T. & 17,1 & 23,5 & 30,7 \\
\hline & & & & 16,2 & 19,7 & 27,8 \\
\hline \multirow{2}{*}{\multicolumn{3}{|c|}{ Durchschnittswert }} & P. T. & 15,9 & 20,1 & 23,8 \\
\hline & & & $\mathrm{k}$. & 13,8 & 17,2 & 22,0 \\
\hline
\end{tabular}

Was die Korrelation zwischen dem Hypophysen- und Thyreoideahormon und dem Stoffwechsel des Organisnus anbelangt, so hat $\mathrm{M} \mathrm{cK}$ in l a $\mathrm{y}^{15)}$ experimentell nachgewiesen, dass der Schilddrisen- und II ypophysenhinterlappenextrakt auf den Stoffwecbsel synergetisch wirken. Z loc $z$ ow e ${ }^{16)}$ hat bemerkt, dass sich die Wirkung des Hypophysenhinterlappenextrakts auf den Stoffwechsel durch vorherige liingere Einnahme von Schilddrüsenprïparaten nicht beeinflussen lïsst. $\mathrm{Od}$ a i $\mathrm{a}^{17}$ iusserte sich dahin, dass die durch Pituitrin herrorgerufenen Verïnderungen des Gaswechsels und Minutenvolums beim $\mathrm{H}_{y}$ perthyreoidismus von denen der Norm nicht besonders verschieden sind, aber bei Athyreoidismus beide durch weit kleinere Mengen Pituitrin vermindert werden als in der Norm oder bei Hyperthyreoidismus. Ferner ninmt $\mathrm{A} \mathrm{hlg} \mathrm{ren}^{2}$ ) an, dass Pituitrin und Thyroxin auf die Gewebsoxydation bald antagonistiseh, bald synergetisch wirken.

15) McKinlay, Arch. Int. Med, 1921, 28, 703.

16) Zloczower, Zeitschr. f. d. ges. exp. Med., 1923, 37, 68.

17) Odaira, Tohoku Journ. Exp. Med, 1925, 6, 325. 
In meiner früheren Mitteilung ${ }^{14)}$ habe ich nachgewiesen, dass Pituglandol bei alleiniger Zufügung zum Blut inmer, sowohl bei grösseren als auch bei kleineren Dosen, seinen Sauerstoffverbrauch herabsetzt, während Thyreoglandol in beiden Dosen ihn fördert. Also zeigen beide Hormone in Bezug auf die Blutzellatmung eine antagonistische Beziehung gegeneinander. Wirken sie auch bei kombinierter Anwendung in entgegengesetztem Sinne zueinander ebenso, wie dies bei alleiniger Anwendung jedes der beiden der Fall ist, so müsste es sich beim Gesamtresultat nur um die Differenz der Plus- oder Minuswirkung handeln, also um die Wirkungsgrösse, welche dem Rest nach der Neutralisation entspricht, somit wird nur sehr geringfügige Förderung oder Hemmung hervorgerufen. Aber, wie die oben beschriebenen Tersuche zeigen, ergibt sich bei gleichzeitigem Zusatz beider Hormone zum Blut sowohl in grösseren als auch in kleineren Dosen immer eine erhöhte Sanerstoffzehrung des Bluts. Es hat also den Anschein, als ob sich hier nur die Wirkung des Thyreoglandols geltend macht.

Zusammenfassung: Auf die Blutzellatmung in vitro wirken das Schilddrüsen- und Hypophysenhinterlappenhormon, jedes für sich allein appliziert, in entgegengesetztem Sinne, während bei ihrem gleichzeitigen Zusatz zum Blut hauptsächlich nur ersteres seine Wirkung zu entfalten scheint, aber nicht letzteres.

\section{Kombinierte Wirkung von Insulin und Pituglandol.}

Von vielen Autoren werden Insulin und Pituitrin als Antagonisten bezeichnet, so in bezug auf Beeinflussung des Kohlehydratstoffwechsels ron $\mathrm{Dale}$ und $\mathrm{Burn}{ }^{18}$, Joachimoglu und Metz, Kitamura, ${ }^{19}$ Voegtlin, Thompson und Dunn, ${ }^{20}$ Lawrence und Hewlett,21) Moehlig und Ainsle e'2) und Vollmer und Serebrijski,23) beim Fettstoffwechsel ron Coope, ${ }^{2+}$ beim Wasserhaushalt ron Vollmer und Serebrijski, ${ }^{23}$, Adlersberg ${ }^{25}$, und Koref und Mautner. ${ }^{20)}$ Nur Ablgren betonte, dass man bei gleichzeitigem Zusatz ron Insulin und Pituitrin eine addierte fördernde Wirkung auf die Gewebsatmung erhält. $C_{m}$ zu erkennen, was für eine Einwirkung sich auf die Sauerstoff-

18) Dale und Burn, Journ. Physiol, 1924, 59, 164.

19) Joachimoglu und Netz, Deutsch. med. Wochenschr., 1924, 1788. 137.

20) Voegtlin, Thompson und Dunn, Journ. Pharm. Exp. Therapeut, 1925, 25,

21) Lawrence und II w lett, Brit. Med. Journ., 1925, 998.

22) Moeblig und Ainslee, Journ. Americ. Med. Ass., 1925, 84, 1398.

23) Vollmer und Serebrijski, Biochem. Zeitschr., 1925, 158, 366.

24) Coope, Journ. Physiol., 1925, 60, 92.

25) Adlersberg, Biochem. Zeitschr., 1926, 169, 129.

26) Koref und M a u tner, Arch. f. exp. Path. u. Pharm., 1926, 113, 124. 
atmung des Bluts bei gleichzeitiger Zufigung der beiden Itormone zum Blut ergibt, habe ich folgende Versuche angestellt:

Es wurde Pituglandol (Roche) und Insulin (Toronto) in relativ grossen Dosen (ron jedem $0,075 \mathrm{ccm}$ ) gleichzeitig $1 \mathrm{ccm}$ Blut zugesetzt und der Sauerstoffverbrauch des Bluts jede dritte Stunde gemessen, was das in Tab. 18 verzeichnete Resultat ergab. Dass Insulin, in dieser Dose für sich allein appliziert, den Sauerstoffverbrauch des Bluts deutlich steigert, ihn Pituclandol aber ein wenig herabsetzt, wurde in meiner vorigen Mitteilung ${ }^{14)}$ erwähnt. Bei kombinierter Anwendung beider Inkrete ist, wie Tab. 18 zeigt, der Sauerstoffkonsum des Bluts in jeder Stunde in allen Fällen kaum

Tabelle 18.

Der Eintlus von Insulin + Pituglandol auf den Sauerstofverbrauch des Blutes.

(1 cm Blut : 0,075 cm Insulin $+0,075 \mathrm{ccm}$ Pituglandol)

I. P. = Blut mit Insulin + Pituglandol. K. $=$ Kontrolle.

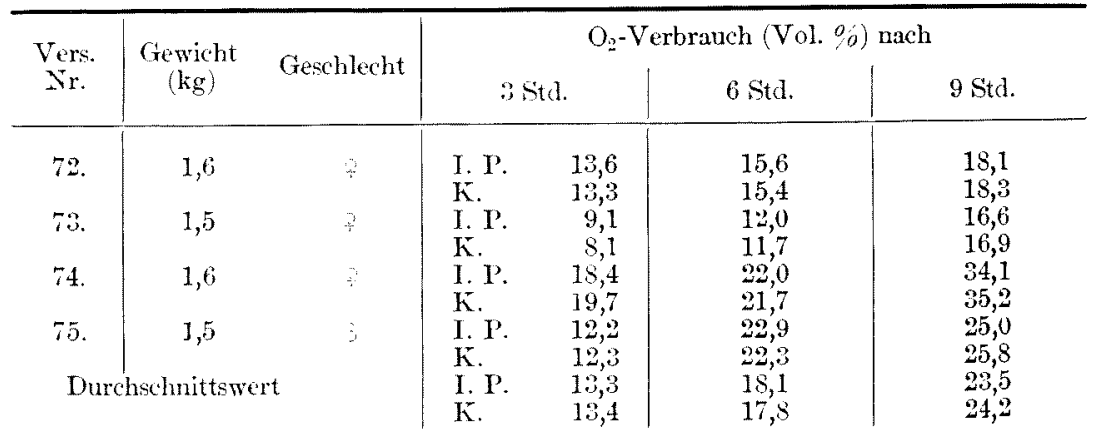

verschieden von dem bei der Kontrolle, was wohl darauf beruht, dass die entgegengesetzte Wirkung jedes einzeluen der beiden Hormone gegenseitig aufgehoben wird. Also kann man annehmen, dass Insulin und Pituglandol anf den Sauerstoffverbrauch des Bluts in relativ grossen Dosen antagonistisch wirken.

Werden sie weiter in relativ kleiner Menge dem Blut gleichzeitig zugefügt $(0,005 \mathrm{~cm}$ Insulin und $0,005 \mathrm{~cm}$ Pituglandol : $1 \mathrm{ccm}$ Blut), so ergibt sich auch ein interessantes Resultat, das in Tab. 19 wiedergegeben ist.

Bei jeweils alleiniger Insulin- oder Pituglandolapplikation in solcher Dose zum Blut wirktersteres auf seinen Saucrstoffverbrauch steigernd, aber letzteres horabsetzend, während sie bei gleichzeitiger Anwendung, wie aus Tab. 19 ersichtlich, ihn kaum beeinflussen, so dass hier beide Hormone in ihrer Wirkung durch einander neutralisiert werden. 
Tabelle 19.

Der Einfluss von Insulin + Pituglandol auf den Sauerstoffverbrauch des Blutes.

(1 ccm Blut : 0,005 $\mathrm{cm}$ Insulin $+0,005 \mathrm{ccm}$ Pituglandol)

I. P. Blut mit Insulin + Pituglandol. $\mathbf{K}$. $=\mathrm{K}$ ontrolle.

\begin{tabular}{|c|c|c|c|c|c|c|}
\hline \multirow{2}{*}{$\begin{array}{l}\text { Vers. } \\
\text { Nr. }\end{array}$} & \multirow{2}{*}{$\begin{array}{l}\text { Gewicht } \\
\text { (kg) }\end{array}$} & \multirow{2}{*}{ Geschlecht } & \multicolumn{4}{|c|}{$\mathrm{O}_{2}$-Verbrauch (Vol. \%) nach } \\
\hline & & & \multicolumn{2}{|c|}{$3 \mathrm{Std}$} & $6 \mathrm{std}$. & $9 \mathrm{std}$. \\
\hline 76. & 1,7 & q & I. P. & 12,9 & 15,1 & 17,0 \\
\hline \multirow[t]{2}{*}{77.} & 1,8 & $\hat{\mathrm{S}}$ & I. $\mathbf{P}$ & 12,1 & 16,6 & $\begin{array}{l}16,0 \\
82,3\end{array}$ \\
\hline & & & $\mathrm{K}$. & 12,9 & 16,8 & 21,8 \\
\hline \multirow[t]{2}{*}{78.} & 1,9 & q & I. $\mathrm{P}$ & 13,6 & 17,5 & 21,4 \\
\hline & & & & 13,2 & 17,3 & 21,8 \\
\hline \multirow[t]{2}{*}{79.} & 2,0 & 9 & I. $\mathbf{P}$ & 17,0 & 19,7 & 27,2 \\
\hline & & & & 16,2 & 19,7 & 27,8 \\
\hline \multicolumn{3}{|c|}{ Durchschnittswert } & I. $P$. & 13,9 & 17,3 & 22,0 \\
\hline & & & K. & 13,8 & 17,3 & 22,0 \\
\hline
\end{tabular}

Wie schon erörtert, behaupteten viele Autoren, dass die Hypophyse und das Pankreas im Gesamtorganismus reine Antagonisten für verschiedene Organwirkungen seien. Dass sich auch in Bezug auf die Sauerstoffatmung der isolierten überlebenden Erythrozyten ein gleiches Verhalten zeigt, ist $\mathrm{m}$. E. von Interesse.

Zusammenfassung: Bei gleichzeitigem Zusatz von Insulin und Pituglandol zum Blut lässt sich sein Sauerstoffverbrauch weder erhöhen noch herabsetzen. Beide Hormone wirken nicht nur auf den Gesamtorganismus, sondern auch auf die Sauerstoffatmung der isolierten überlebenden Erythrozyten antagonistisch.

\section{Schluss.}

Bei der Übersicht der oben erwühnten Ergebnisse gelange ich zu folgenden Schlüssen :

1. Bei gleichzeitigem Zusatz von Atropin und Insulin zum defibrinierten Blut wirken sie auf seinen Sauerstoffverbrauch in vitro je nach ihrer Menge in verschiedener Weise; in relativ grossen Dosen fördernd, dagegen in kleineren hemmend. Da sich die Blutatmung in vitro durch Atropin in grösseren Mengen ein wenig beschleunigen lässt, so wird dic Insulinförderung durch Atropinzusatz nicht aufgehoben, aber bei kleinen Dosen von Atropin macht sich eine Hemmung geltend, die die beschleunigende Insulinwirkung überwindet.

2. Wird das Blut in vitro gleichzeitig mit Insulin und Pilokarpin 
versetzt, so zeigt sich, da letzteres, allein in grossen Dosen appliziert, unwirksam ist, nur die fördernde Wirkung des ersteren auf den Sauerstoffkonsum des Bluts. Bei kleiner Dosierung, bei der jedes der beiden allein tördernd wirkt, weisen sie kombiniert auch Förderung auf, deren Stärke aber nicht der algebraischen Summe der Einzelwirkungen gleichkommt.

3. Bei gleichzeitiger Zufügung von Adrenalin und Atropin in relativ grossen Dosen zum Blut lässt seine Atmung kaum beeinflussen, aber in relativ kleincn wird die Blutatmung in vitro herabgesetzt, was wohl daher rührt, dass die Wirkung durch Atropin aufgehoben wird.

4. Bei kombinierter Anwendung von Adrenalin und Pilokarpin wird der Sanerstoffverbrauch des Bluts kaum verändert; sie scheinen auf die Blutzellatmung antagonistisch zueinander zu wirken.

5. Die kombinierte Wirkung von Insulin und Adrenalin auf den Sauerstoffverbrauch des Bluts, sowohl in grösseren als auch in kleineren Dosen, ist immer eine beschleunigende. Sie beide wirken also auf die Blutatmung in vitro in grossen Dosen antagonistisch, aber in kleinen synergetisch.

6. Bei gleichzeitigem Zusatz von Ergotamin und Adrenalin zum Blut lïsst sich seine Saucrstoffutmung weder verstïrken noch hemmen. Also üben sie beide auch auf die Blutatmung in vitro einc entgegengesetzte Wirkung ans.

7. Wenn man Ergotamin und Atropin gleichzeitig dem Blut in vitro zufügt, so wird die Blutatmung herabgesetzt, indem nur die Atropinwirkung zum Vorschein kommt.

8. Eine relativ gresse Menge von Ergotamin hebt die Pilokarpinwirkung auf, die Blutatmung lässt sich kaum beschleunigen, hingegen scheint der Effekt des Ergotamins in kleiner Menge durch die Pilokarpinwirkung ausgeschaltet zu werden, da der Sauerstoffkonsum des Bluts erheblich Förderung crfährt.

9. Lässt man das Schilddrüsen- und Hypophysenhinterlappenhormon jedes allein auf die Blutatmung in vitro einwirken, so ergibt sich bei beiden ein entgegengesetztes Resultat, während, wenn beide gleichzeitig hinzugefügt werden, hauptsïchlich nur die Wirkung des ersteren aufzutreten scheint.

10. Insulin und Pituglandol wirken auch auf die Sauerstoffatmung des Bluts in vitro antagonistisch zueinander, sie wird bei gleichzeitigem Zusatz beider Inkrete zum Blute so gut wie nicht beeinflusst.

11. Fasst man die korrelative Wirkung der oben geschilderten Hormone und vegetativen Nervengifte auf die Sauerstoffatmung des Bluts zu- 
Wirkung der Inkrete u. Vegetativnervengifte auf $\mathrm{O}_{2}$-Verbrauch d. Bluts 353 sammen, so lässt sich erkennen, dass sie im grossen und ganzen ein ähnliches Verhalten wie gegen den Gesamtorganismus aufweisen. 